\title{
A Hierarchy of Aryloxide Deprotection by Boron Tribromide
}

\author{
Sreenivas Punna, Stéphane Meunier and M. G. Finn* \\ Department of Chemistry and The Skaggs Institute for Chemical Biology, The Scripps Research \\ Institute, 10550 N. Torrey Pines Rd., La Jolla, CA 92037 USA
}

\section{Supporting Information}

General: ${ }^{1} \mathrm{H}$ and ${ }^{13} \mathrm{C}$ NMR spectra were recorded on Bruker DRX-500, AMX-400, or Varian Mercury 200 spectrometer, using $\mathrm{CDCl}_{3}$ or $\mathrm{CD}_{3} \mathrm{OD}$ as solvents. Mass spectra were obtained on Agilent $1100 \mathrm{LC} / \mathrm{MS}$ spectrometer (model G1946A), Agilent-ESI-TOF, or Agilent 5973N GC/MS. Melting points were measured in a Mel-Temp II capillary melting point apparatus and are uncorrected. Infrared spectra were recorded on a MIDAC EM200 instrument with horizontal attenuated total reflectance accessory from Pike Instruments or in $\mathrm{KBr}$ pellets. Column chromatography was performed on EM Science silica gel, $60 \AA$ and 40-63 $\mu \mathrm{m}$. TLC analysis was facilitated by the use of phosphomolybdic acid or $\mathrm{KMnO}_{4} / \mathrm{H}_{2} \mathrm{O}$ stains in addition to UV light with fluorescent-indicating plates. All commercially available reagents were purchased from Aldrich or Acros or TCI-America. Reactions requiring anhydrous conditions were performed under nitrogen. Anhydrous sodium sulfate was used for drying solutions.

Note: Compound numbers used here correspond to the entry numbers in Table 1 of the text, "a" representing the starting materials and " $\mathrm{b}$ " the products of treatment with $\mathrm{BBr}_{3}$.

\section{General propargylation procedure:}

A mixture of the corresponding phenol $(6.57 \mathrm{mmol})$, anhydrous potassium carbonate $(19.71 \mathrm{mmol})$ and propargyl bromide $(80 \%$ in toluene, $7.88 \mathrm{mmol})$ in acetone $(10 \mathrm{~mL})$ was stirred overnight at room temperature. The solution was filtered, water was added, and the product was extracted three times with ether. The combined organic layers were dried over sodium sulfate and evaporated under reduced pressure. The residue was purified by column chromatography to afford the desired propargyl ether. The identities of compounds $1 \mathrm{a},{ }^{1} \mathbf{3 a},{ }^{2} \mathbf{5 a},{ }^{3} \mathbf{6 a},{ }^{2} 7 \mathbf{a},{ }^{4}$ and $9 \mathbf{a}^{5}$ were confirmed by comparison to known spectra.

1-Methoxy-3-prop-2-ynyloxy-benzene (4a): the general protection procedure was applied to 3methoxyphenol to afford $4 \mathbf{a}(99 \%)$ as a colorless liquid. ${ }^{1} \mathrm{H}$ NMR $\left(\mathrm{CDCl}_{3}\right) \delta 2.53(\mathrm{t}, 1 \mathrm{H},-\mathrm{CCH}) ; 3.80$ (s, $\left.3 \mathrm{H},-\mathrm{OCH}_{3}\right) ; 4.68\left(\mathrm{~d}, J=2.61 \mathrm{~Hz}, 2 \mathrm{H},-\mathrm{CH}_{2}\right)$; 6.52-6.64 (m, 3H, Aryl); 7.14-7.30 (m, $1 \mathrm{H}$, Aryl $) ;{ }^{13} \mathrm{C}$ NMR $\left(\mathrm{CDCl}_{3}\right) \delta 55.0,55.5,75.2,78.2,101.2,106.6,106.9,129.6,158.5,160.5$; IR (thin film, $\left.\mathrm{cm}^{-1}\right): 3312$ (HCC-), 3002, 2954, 2833, 2122 (alkyne), 1594, 1280, 1260, 1195, 1151, 1047; GCMS m/z 162 (M), 161, 147,95 .

2-(3-Prop-2-ynyloxy-benzyloxy)-tetrahydro-pyran (8a): To a solution of 3-(2propynyloxy)benzenemethanol $(200 \mathrm{mg}, 1.23 \mathrm{mmol})^{6}$ in $\mathrm{CH}{ }_{2} \mathrm{Cl}_{2}(10 \mathrm{~mL})$ was added 3,4-dihydro-2 $\mathrm{H}$ pyran $(0.17 \mathrm{~mL}, 1.85 \mathrm{mmol})$ and $p$-toluenesulfonic acid $(20 \mathrm{mg}, 0.1 \mathrm{mmol})$. The mixture was stirred overnight at room temperature, and then solid sodium bicarbonate was added to neutralize the acid. After filtration of the solid and evaporation under reduced pressure, the residue was purified by column chromatography (10\% ethyl acetate / hexane) to afford $110 \mathrm{mg}(36 \%)$ of $\mathbf{8 a}$ as a colorless liquid. ${ }^{1} \mathrm{H}$ NMR $\left(\mathrm{CDCl}_{3} ; 200 \mathrm{MHz}\right): \delta 1.40-2.10(\mathrm{~m}, 6 \mathrm{H}, \mathrm{THP}) ; 2.52(\mathrm{t}, J=2.40 \mathrm{~Hz},-\mathrm{CCH}) ; 3.45-3.65(\mathrm{~m}, 1 \mathrm{H}$, THP); 3.85-4.05 (m, 1H, THP); 4.49 (d, $\left.J=12.4 \mathrm{~Hz}, 1 \mathrm{H}, \mathrm{Ar}-\mathrm{CH}_{2}-\mathrm{OTHP}\right)$; 4.65-4.75 (m, 3H, THP, $-\mathrm{CH}_{2}-$

\footnotetext{
${ }^{1}$ Ishii, H.;Ishikawa, T.; Takeda, S.; Ueki, S; Masahiro, S. Chem. Pharm. Bull. 1992, 40, 1148-1153.

${ }^{2}$ Pal, M.; Parasuraman, K.; Yleswarapu, K. R. Org. Lett. 2003, 5, 349-352.

${ }^{3}$ Atwal, K. S.; Grover, G. J.; Ferrara, F. N.; Ahmed, S. Z.; Sleph, P. G.; Dzwonczyk, S.; Normandin, D. E. J. Med. Chem. 1995, 38, 1966-1973.

${ }^{4}$ Ishikawa, T.; Nagai, K.; Ohkubo, N.; Ishii, H. Heterocycles, 1994, 39, 371-380.

${ }^{5}$ Schvartzapel, A. J.; Zhong, L.; Docampo, R.; Rodriguez, J. B.; Gros, E. G. J. Med. Chem. 1997, 40, 23142322.

${ }^{6}$ Kanamathareddy, S.; Gutsche, C. D. J. Org. Chem. 1996, 61, 2511-2516.
} 
); 4.77 (d, $J=12.4 \mathrm{~Hz}, 1 \mathrm{H}$, Ar- $\mathrm{CH}_{2}$-OTHP); 6.85-7.10 (m, 3H, Aryl); 7.20-7.35 (m, 1H, Aryl); ${ }^{13} \mathrm{C}$ NMR $\left(\mathrm{CDCl}_{3}\right) \delta 19.4,25.6,30.7,55.9,62.2,68.6,75.6,78.7,97.8,114.2,121.0,129.5,140.2,157.8$; IR (thin film, $\mathrm{cm}^{-1}$ ): 3289 (HCC-), 2935, 2862, 2118 (alkyne), 1446, 1341, 1264, 975, 902, 866; ESMS $\mathrm{m} / \mathrm{z}$ (relative intensity) $269(\mathrm{M}+\mathrm{Na})^{+}$(62), 121 (100); HRMS calcd for $\mathrm{C}_{15} \mathrm{H}_{18} \mathrm{O}_{3} \mathrm{Na}$ 269.1154, found 269.1058.

1-Allyloxy-4-prop-2-ynyloxy-benzene (10a): the general protection procedure was applied to 4allyloxyphenol, ${ }^{7}$ to afford $10 a(75 \%)$ as a white solid. $\mathrm{Mp} 47-48{ }^{\circ} \mathrm{C} ;{ }^{1} \mathrm{H} \mathrm{NMR}\left(\mathrm{CDCl}_{3} ; 200 \mathrm{MHz}\right): \delta 2.52$ (t, $J=2.4 \mathrm{~Hz}, 1 \mathrm{H},-\mathrm{CCH}) ; 4.50\left(\mathrm{~d}, J=5.4 \mathrm{~Hz}, 2 \mathrm{H},-\mathrm{CH}_{2}-\right) ; 4.64$ (d, $\left.J=2.4 \mathrm{~Hz}, 2 \mathrm{H},-\mathrm{CH}_{2}-\right) ; 5.20-5.50(\mathrm{~m}$, $\left.2 \mathrm{H},-\mathrm{CH}=\mathrm{CH}_{2}\right) ; 5.95-6.20\left(\mathrm{~m}, 1 \mathrm{H},-\mathrm{CH}=\mathrm{CH}_{2}\right) ; 6.80-7.00(\mathrm{~m}, 4 \mathrm{H}, \mathrm{Aryl}) ;{ }^{13} \mathrm{C} \mathrm{NMR}\left(\mathrm{CDCl}_{3}\right) \delta 56.3,69.1$, 75.0, 78.6, 115.3, 115.8, 117.2, 133.2, 151.5, 153.2; IR (KBr, cm $\left.{ }^{-1}\right): 3273$ (HCC-), 3023 (-C=C-H), 2990, 2914, 2858, 2133 (alkyne), 2045, 1860, 1630 (-C=C-), 1505, 1215, 1020, 825, 795; GCMS m/z 188 (M), 147, 91; Anal. Calcd for $\mathrm{C}_{12} \mathrm{H}_{12} \mathrm{O}_{2}:$ C, 76.57; H, 6.43. Found: C, 76.73; H, 6.47.

1-Benzyloxy-4-prop-2-ynyloxy-benzene (11a): the general protection procedure was applied to 4(benzyloxy)phenol to afford 11a (99\%) as a white solid. Mp $45-46{ }^{\circ} \mathrm{C} ;{ }^{1} \mathrm{H}$ NMR $\left(\mathrm{CDCl}_{3} ; 200 \mathrm{MHz}\right)$ : $\delta$ $2.52(\mathrm{t}, J=2.4 \mathrm{~Hz} ; 1 \mathrm{H},-\mathrm{CCH}) ; 4.65\left(\mathrm{~d}, J=2.4 \mathrm{~Hz}, 2 \mathrm{H},-\mathrm{CH}_{2}-\mathrm{CC}\right) ; 5.04$ (s, 2H, - $\left.\mathrm{OCH}_{2}-\mathrm{Ar}\right) ; 6.94$ (s, 4H, $\left.\mathrm{O}-\mathrm{C}_{6} \mathrm{H}_{4}-\mathrm{O}-\right) ; 7.25-7.55(\mathrm{~m}, 5 \mathrm{H}) ;{ }^{13} \mathrm{C}$ NMR $\left(\mathrm{CDCl}_{3}\right) \delta$ 56.2, 70.3, 75.2, 78.7, 115.2, 115.8, 127.2, 127.7, 128.3, 137.0, 151.6, 153.4; IR (KBr, cm ${ }^{-1}$ ): 3280 (HCC-), 2902, 2861, 2117 (alkyne), 1847, 1509, 1453, $1377,1227,1034,1010,813$; ESMS $m / z$ (relative intensity) $261(\mathrm{M}+\mathrm{Na})^{+}(50), 105(100)$; Anal. Calcd for $\mathrm{C}_{16} \mathrm{H}_{14} \mathrm{O}_{2}: \mathrm{C}, 80.65 ; \mathrm{H}, 5.92$. Found: $\mathrm{C}, 81.01 ; \mathrm{H}, 6.05$.

4-Methoxy-benzoic acid prop-2-ynyl ester (12a): To a solution of 4-methoxybenzoic acid (1.0 g, 6.57 $\mathrm{mmol})$ in $\mathrm{CH}_{2} \mathrm{Cl}_{2}(20 \mathrm{~mL})$ was added propargyl alcohol $(0.38 \mathrm{~mL}, 6.57 \mathrm{mmol})$, DMAP $(80 \mathrm{mg}, 0.66$ $\mathrm{mmol})$ and DCC $(2.0 \mathrm{~g}, 9.85 \mathrm{mmol})$. The mixture was stirred for 10 hours at room temperature, filtered, and evaporated under reduced pressure. The residue was purified by column chromatography $(20 \%$ ethyl acetate / hexane) to afford $762 \mathrm{mg}(63 \%)$ of $\mathbf{1 2 a}$ as a white solid. Mp 40-41 ${ }^{\circ} \mathrm{C} ;{ }^{1} \mathrm{H}$ NMR $\left(\mathrm{CDCl}_{3} ; 200\right.$ $\mathrm{MHz}): \delta 2.48-2.54(\mathrm{~m}, 1 \mathrm{H},-\mathrm{CCH}) ; 3.82\left(\mathrm{~s},-\mathrm{OCH}_{3}\right) ; 4.84-4.92\left(\mathrm{~m}, 2 \mathrm{H},-\mathrm{OCH}_{2}-\mathrm{CC}\right) ; 6.89(\mathrm{~d}, J=9.20 \mathrm{~Hz}$, $2 \mathrm{H}$, Aryl); $7.98(\mathrm{~d}, J=9.20 \mathrm{~Hz}, 2 \mathrm{H}, \mathrm{Aryl}) ;{ }^{13} \mathrm{C} \mathrm{NMR}\left(\mathrm{CDCl}_{3}\right) \delta 52.3,55.6,75.1,78.2,113.8,121.9$, 132.0, 163.8, 165.4; IR (KBr, cm ${ }^{-1}$ ): 3240 (HCC-), 2934, 2841, 2121 (alkyne), 1710 (-C=O), 1602, 1509, 1264, 1167, 1095, 1022, 845, 786; GCMS m/z 190 (M).

Terephthalic acid 1-methyl ester 4-prop-2-ynyl ester (13a): To a solution of mono-methyl terephthalate $(0.5 \mathrm{~g}, 2.77 \mathrm{mmol})$ in DMF $(6 \mathrm{~mL})$ was added potassium carbonate $(1.15 \mathrm{~g}, 8.32 \mathrm{mmol})$ and propargyl bromide $(80 \%$ in toluene, $0.62 \mathrm{~mL}, 5.55 \mathrm{mmol})$. The mixture was stirred overnight at $70^{\circ} \mathrm{C}$. A saturated solution of ammonium chloride was added, and the mixture was extracted three times with ethyl acetate. The combined organic layers were dried over anhydrous $\mathrm{Na}_{2} \mathrm{SO}_{4}$ and evaporated under reduced pressure. The residue was purified by column chromatography (10\% ethyl acetate / hexane) to afford 595 mg (98 \%) of 13a as a white solid. Mp 81-82 ${ }^{\circ} \mathrm{C} ;{ }^{1} \mathrm{H}$ NMR (CDCl $\left.; 200 \mathrm{MHz}\right): \delta 2.54(\mathrm{t}, J=2.2 \mathrm{~Hz}, 1 \mathrm{H}$, $\mathrm{CCH}) ; 3.94$ (s, $\left.3 \mathrm{H},-\mathrm{OCH}_{3}\right) ; 4.94\left(\mathrm{~d}, J=2.2 \mathrm{~Hz},-\mathrm{CH}_{2}-\mathrm{CC}\right) ; 8.11$ (s, $\left.4 \mathrm{H}, \mathrm{Aryl}\right) ;{ }^{13} \mathrm{C} \mathrm{NMR}\left(\mathrm{CDCl}_{3}\right) \delta 52.1$, $52.5,75.1,77.1,129.1,129.20,129.23,129.39,132.7,132.8,164.5,165.7$, IR (KBr, cm $\left.{ }^{-1}\right): 3253$ (HCC-), 2963, 2126 (alkyne), 1720 (-C=O), 1438, 1406, 1276, 1111, 955, 721, 673; GCMS $m / z 218$ (M), 63, 135; Anal. Calcd for $\mathrm{C}_{12} \mathrm{H}_{10} \mathrm{O}_{4}$ : C, 66.05; H, 4.62. Found: C, 66.01; H, 4.69.

4-Methoxy- $\boldsymbol{N}$-prop-2-ynyl-benzamide (14a): To a solution of 4-methoxybenzoic acid (1.0 g, 6.57 $\mathrm{mmol})$ in $\mathrm{CH}_{2} \mathrm{Cl}_{2}(20 \mathrm{~mL})$ was added propargylamine $(0.42 \mathrm{~mL}, 6.57 \mathrm{mmol})$, DMAP $(80 \mathrm{mg}, 0.66 \mathrm{mmol})$ and DCC $(2.0 \mathrm{~g}, 9.85 \mathrm{mmol})$. The mixture was stirred for 10 hours at room temperature, filtered, and the filtrate was evaporated under reduced pressure. The residue was purified by column chromatography (40 $\%$ ethyl acetate / hexane) to afford $849 \mathrm{mg}(68 \%)$ of $\mathbf{1 4 a}$ as a white solid. Mp 124-125 ${ }^{\circ} \mathrm{C} ;{ }^{1} \mathrm{H}$ NMR $\left(\mathrm{CDCl}_{3} ; 200 \mathrm{MHz}\right): \delta$ 2.25-2.30 (m, 1H, $\left.-\mathrm{CCH}\right) ; 3.83\left(\mathrm{~s}, 1 \mathrm{H},-\mathrm{OCH}_{3}\right) ; 4.15-4.28\left(\mathrm{~m}, 2 \mathrm{H},-\mathrm{NH}-\mathrm{CH}_{2}-\right) ; 6.56$ (brs., $1 \mathrm{H},-\mathrm{NH}-) ; 6.90$ (d, $J=8.80 \mathrm{~Hz}, 2 \mathrm{H}$, Aryl); $7.76(\mathrm{~d}, J=8.80 \mathrm{~Hz}, 2 \mathrm{H}$, Aryl $) ;{ }^{13} \mathrm{C} \mathrm{NMR}\left(\mathrm{CDCl}_{3}\right) \delta$

\footnotetext{
${ }^{7}$ Lee, K. C.; Moon, B. S.; Lee, J. H.; Chung, K. H.; Katzenellenbogen, J. A.; Chi, D. Y. Bioorg. Med. Chem. 2003, 11, 3649-3658.
} 
29.9, 55.6, 71.8, 80.0, 113.9, 126.2, 129.1, 162.5, 166.9; IR (KBr, $\left.\mathrm{cm}^{-1}\right): 3264$ (HCC-), 2930, 2837, 2120 (alkyne), 1892, $1634(-\mathrm{C}=\mathrm{O}), 1494,1409,1300,1258,1179,1030,835,765$; ESMS $\mathrm{m} / z$ (relative intensity) $212(\mathrm{M}+\mathrm{Na})^{+}(90), 190(\mathrm{M}+1)^{+}(100)$; Anal. Calcd for $\mathrm{C}_{11} \mathrm{H}_{11} \mathrm{NO}_{2}: \mathrm{C}, 69.83 ; \mathrm{H}, 5.86 ; \mathrm{N}, 7.40$. Found: C, 69.68; H, 6.09; N, 7.58.

4-Methoxy- $\boldsymbol{N}$-methyl- $\boldsymbol{N}$-prop-2-ynyl-benzamide (15a): To a solution of $p$-anisic acid acid $(0.5 \mathrm{~g}, 3.28$ $\mathrm{mmol})$ in $\mathrm{CH}_{2} \mathrm{Cl}_{2}(10 \mathrm{~mL})$ was added $N$-methylpropargylamine $(0.30 \mathrm{~mL}, 3.61 \mathrm{mmol})$ and DCC $(746 \mathrm{mg}$, $3.61 \mathrm{mmol})$. The mixture was stirred overnight at room temperature. DCU was filtered, the filtrate was evaporated under reduced pressure, and the residue was purified by column chromatography (30\% ethyl acetate / hexane) to afford $458 \mathrm{mg}(68 \%)$ of $\mathbf{1 5 a}$ as a pale yellow syrup. ${ }^{1} \mathrm{H}$ NMR $\left(\mathrm{CDCl}_{3} ; 200 \mathrm{MHz}\right): \delta$ 2.27-2.35 (m, $1 \mathrm{H},-\mathrm{CCH}) ; 3.10\left(\mathrm{~s}, 3 \mathrm{H},-\mathrm{NCH}_{3}\right) ; 3.82\left(\mathrm{~s}, 3 \mathrm{H},-\mathrm{OCH}_{3}\right) ; 4.05-4.30\left(\mathrm{~m}, 2 \mathrm{H},-\mathrm{OCH}_{2}-\mathrm{CC}\right) ; 6.90$ $(\mathrm{d}, J=8.8 \mathrm{~Hz}, 2 \mathrm{H}$, Aryl $) ; 7.46\left(\mathrm{~d}, J=8.8 \mathrm{~Hz}, 2 \mathrm{H}\right.$, Aryl); ${ }^{13} \mathrm{C} \mathrm{NMR}\left(\mathrm{CDCl}_{3}+\mathrm{CD}_{3} \mathrm{OD}\right) \delta 32.5,40.2,53.9$, 71.2, 77.0, 112.6, 125.8, 127.8, 160.3, 171.0, 171.06, 172.4; IR (KBr, $\left.\mathrm{cm}^{-1}\right): 3284$ (HCC-), 2926, 2845, 2117 (alkyne), $1622(-\mathrm{C}=\mathrm{O}), 1389,1296,1252,1172,1066,1026,837,769 ;$ ESMS $\mathrm{m} / z$ (relative intensity) $226(\mathrm{M}+\mathrm{Na})^{+}(78), 204(\mathrm{M}+1)^{+}(100)$; HRMS calcd for $\mathrm{C}_{12} \mathrm{H}_{13} \mathrm{NO}_{2}$ 204.1019, found 204.1015.

$N$-(3,5-Bis-prop-2-ynyloxy-benzyl)-2-chloro-terephthalamic acid methyl ester (16a): Compound 16a was prepared as part of an independent study and its detailed synthesis will be reported separately. White solid; ${ }^{1} \mathrm{H}$ NMR $\left(\mathrm{CDCl}_{3} ; 200 \mathrm{MHz}\right): \delta 2.48-2.54(\mathrm{~m}, 2 \mathrm{H},-\mathrm{CCH}) ; 3.93\left(\mathrm{~s}, 3 \mathrm{H},-\mathrm{OCH}_{3}\right) ; 4.53(\mathrm{~d}, J=$ $\left.5.4 \mathrm{~Hz}, 2 \mathrm{H},-\mathrm{CH}_{2}-\mathrm{N}\right)$; 4.60-4.67 (m, 4H, $\left.-\mathrm{CH}_{2}-\mathrm{CC}\right) ; 6.50-6.60(\mathrm{~m}, 3 \mathrm{H}$, Aryl); $6.83(\mathrm{t}, J=6.1 \mathrm{~Hz}, 1 \mathrm{H},-$ $\mathrm{NH}-)$; 7.64-7.72 (m, 1H, Aryl); 7.78-7.88 (m, 2H, Aryl). IR (KBr, cm $\left.{ }^{-1}\right): 3261$ (HCC-), 2951, 2118 (alkyne), 1720 (-C=O), 1627, 1587, 1466, 1289, 1245, 1156, 1044, 826, 754.

(2-Prop-2-ynyloxy-ethyl)-benzene (17a): To a solution of phenethyl alcohol (1.0 g, $8.18 \mathrm{mmol})$ in THF $(15 \mathrm{~mL})$ at $0^{\circ} \mathrm{C}$ was added sodium hydride $(0.36 \mathrm{~g}, 8.99 \mathrm{mmol})$ followed by propargyl bromide $(80 \%$ in toluene, $1.37 \mathrm{~mL}, 12.27 \mathrm{mmol}$ ) after $5 \mathrm{~min}$. The mixture was stirred overnight at room temperature. Water was added, and extracted three times with ether. The combined organic layers were dried over anhydrous $\mathrm{Na}_{2} \mathrm{SO}_{4}$, and evaporated under reduced pressure. The residue was purified by column chromatography (10 $\%$ ethyl acetate / hexane) to afford $917 \mathrm{mg}(70 \%)$ of $\mathbf{1 7 a}$ as a yellow syrup. ${ }^{1} \mathrm{H}$ NMR $\left(\mathrm{CDCl}_{3} ; 200 \mathrm{MHz}\right)$ :

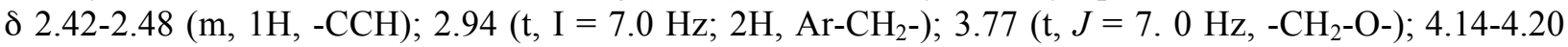
$\left(\mathrm{m}, 2 \mathrm{H},-\mathrm{CH}_{2}-\mathrm{CCH}\right) ; 7.20-7.40$ (m, 5H, Aryl); ${ }^{13} \mathrm{C} \mathrm{NMR}\left(\mathrm{CDCl}_{3}\right) \delta 35.5,57.5,70.2,74.1,79.4,125.8$, 127.9, 128.4, 138.2; IR (KBr, cm ${ }^{-1}$ ): 3313 (HC-), 2946, 2855, 2121 (alkyne), 1558, 1357, 1256, 1095; ESMS $m / z$ (relative intensity) $183(\mathrm{M}+\mathrm{Na})^{+}(38), 105(100)$.

1-But-2-ynyloxy-4-methoxy-benzene (18a): To a solution of $p$-methoxyphenol $(1.0 \mathrm{~g}, 8.05 \mathrm{mmol})$ in acetone $(10 \mathrm{~mL})$ was added potassium carbonate $(3.34 \mathrm{~g}, 24.15 \mathrm{mmol})$ followed by 1-bromo-2-butyne $(0.78 \mathrm{~mL}, 8.86 \mathrm{mmol})$. The mixture was stirred for $6 \mathrm{~h}$ at $60^{\circ} \mathrm{C}$. Water was added, and the mixture was extracted three times with ether. The combined organic layers were dried over anhydrous $\mathrm{Na}_{2} \mathrm{SO}_{4}$ and evaporated under reduced pressure. The residue was purified by column chromatography $(5 \%$ ethyl acetate / hexane) to afford 18a (1.42 g, $99 \%)$ as a colorless liquid. ${ }^{1} \mathrm{H}$ NMR $\left(\mathrm{CDCl}_{3} ; 200 \mathrm{MHz}\right): \delta 1.84-$ $1.90\left(\mathrm{~m}, 3 \mathrm{H},-\mathrm{CCH}_{3}\right) ; 3.77\left(\mathrm{~s}, 3 \mathrm{H},-\mathrm{OCH}_{3}\right) ; 4.56-4.64\left(\mathrm{~m}, 2 \mathrm{H},-\mathrm{CH}_{2}-\mathrm{CC}\right) ; 6.80-6.96\left(\mathrm{~m}, 4 \mathrm{H}\right.$, Aryl); ${ }^{13} \mathrm{C}$ NMR $\left(\mathrm{CDCl}_{3}\right) \delta 55.6,57.1,74.8,83.5,114.7,116.0,152.1,154.4 ; \mathrm{IR}\left(\mathrm{KBr}, \mathrm{cm}^{-1}\right): 3000,2151,2837$, 2226 (alkyne), 1739, 1497, 1441, 1364, 1211, 1042; GCMS m/z 176 (M), 161, 123, 95.

1,3-Dimethyl-2,5-bis-prop-2-ynyloxy-benzene (19a): To a solution of 2,6-dimethylhydroquinone (1.0 g, $7.2 \mathrm{mmol})$ in DMF $(10 \mathrm{~mL})$ was added potassium carbonate $(6.0 \mathrm{~g}, 43.5 \mathrm{mmol})$ followed by propargyl bromide $\left(3.2 \mathrm{~mL}, 22 \mathrm{mmol}, 80 \%\right.$ in toluene). The mixture was stirred for overnight at $80^{\circ} \mathrm{C}$. Water was added, and the mixture was extracted three times with ether. The combined organic layers were dried over anhydrous $\mathrm{Na}_{2} \mathrm{SO}_{4}$ and evaporated under reduced pressure. The residue was purified by column chromatography ( $15 \%$ ethyl acetate / hexane) to afford 19a $(1.1 \mathrm{~g}, 73 \%)$ as white solid. $\mathrm{Mp} 45-46{ }^{\circ} \mathrm{C} ;{ }^{1} \mathrm{H}$ NMR $\left(\mathrm{CDCl}_{3}\right) \delta 2.30$ (s, 6H, Ar- $\left.\mathrm{CH}_{3}\right), 2.54$ (t, $\left.J=2.40 \mathrm{~Hz}, 2 \mathrm{H},-\mathrm{CCH}\right), 4.45,4,61(2 \mathrm{~d}, J=2.40 \mathrm{~Hz}, 4 \mathrm{H}$, $\left.\mathrm{CH}_{2}-\mathrm{CC}\right), 6.62$ (s, 2H, Aryl); ${ }^{13} \mathrm{C}$ NMR $\left(\mathrm{CDCl}_{3}\right) \delta$ 17.1, 56.2, 60.2, 75.4, 75.7, 79.2, 79.8, 109.9, 115.0, 132.3, 150.0, 153.8; IR (KBr, cm $\left.{ }^{-1}\right): 3317$ (HCC-), 3265, 2931, 2874, 2126 (alkyne), 1997, 1606, 1369, 1196, 838; ESMS $m / z$ (relative intensity) $237(\mathrm{M}+\mathrm{Na})^{+}(15), 215(\mathrm{M}+1)^{+}$. 
5-Methoxy-1,3-dimethyl-2-prop-2-ynyloxy-benzene (20a): Compound 19b (the major product obtained from 19a by treatment with one equivalent of $\mathrm{BBr}_{3}$ was alkylated with MeI to afford $20 \mathrm{a}$ (75\% yield) as colorless oil. ${ }^{1} \mathrm{H}$ NMR $\left(\mathrm{CDCl}_{3}\right) \delta 2.35\left(\mathrm{~s}, 6 \mathrm{H}, \mathrm{Ar}-\mathrm{CH}_{3}\right), 2.58(\mathrm{t}, J=2.0 \mathrm{~Hz}, 1 \mathrm{H},-\mathrm{CCH}), 3.76(\mathrm{~s}, 3 \mathrm{H}, \mathrm{Ar}-$ $\left.\mathrm{OCH}_{3}\right), 4.70\left(\mathrm{~d}, J=2.0 \mathrm{~Hz}, 2 \mathrm{H},-\mathrm{CH}_{2}-\right), 6.70(\mathrm{~s}, 2 \mathrm{H}, \mathrm{Aryl}) ;{ }^{13} \mathrm{C} \mathrm{NMR}\left(\mathrm{CDCl}_{3}\right) \delta 16.2,55.8,59.7,75.0$, 78.7, 114.6, 131.6, 151.3, 153.1; IR (thin film, $\mathrm{cm}^{-1}$ ): 3289 (HCC-), 2926, 2862, 2371, 2126 (alkyne), $1598,1486,1321,1220,1180,1152,1055,858$; ESMS $m / z$ (relative intensity) $213(\mathrm{M}+\mathrm{Na})^{+}(20), 189$ (40), $159(25)$.

1,3-Di-tert-butyl-5-methoxy-2-prop-2-ynyloxy-benzene (21a): Compound 21a was prepared using the method described for 19a in $32 \%$ yield $\left(\sim 50 \%\right.$ of starting material recovered) as a colorless liquid. ${ }^{1} \mathrm{H}$ $\operatorname{NMR}\left(\mathrm{CDCl}_{3}\right) \delta 1.46\left(\mathrm{~s}, 18 \mathrm{H},-\mathrm{C}\left(\mathrm{CH}_{3}\right)_{3}\right), 2.53(\mathrm{t}, J=2.40 \mathrm{~Hz},-\mathrm{CCH}), 3.78\left(\mathrm{~s}, 3 \mathrm{H}, \mathrm{Ar}-\mathrm{OCH}_{3}\right), 4.35(\mathrm{~d}, J=$ $2.6 \mathrm{~Hz},-\mathrm{CH}_{2}-\mathrm{CC}$ ), 6.80 (s, 2H, Aryl); IR (thin film, $\mathrm{cm}^{-1}$ ): 3296 (HCC-), 2692, 2910, 2870, 2371, 2129 (alkyne), 1590, 1433, 1356, 1199, 1062, 869, 764; ${ }^{13} \mathrm{C} \mathrm{NMR}\left(\mathrm{CDCl}_{3}\right) \delta$ 29.4, 29.9, 31.8, 35.7, 55.0, 63.3, 74.3, 79.0, 111.7, 144.4, 150.3, 154.6; GCMS m/z 274 (M, low), 235.

General $\mathrm{BBr}_{3}$-mediated propargyl deprotection procedure: To a solution of propargyl ether (1 mmol) in dry $\mathrm{CH}_{2} \mathrm{Cl}_{2}(3 \mathrm{~mL})$ under $\mathrm{N}_{2}$ atmosphere was added $\mathrm{BBr}_{3}\left(1 \mathrm{M}\right.$ in $\left.\mathrm{CH}_{2} \mathrm{Cl}_{2}, 1 \mathrm{mmol}\right)$ dropwise at room temperature. The reaction mixture was monitored by TLC; upon completion, ice-water was added and the mixture was extracted three times with ethyl acetate. The combined organic layers were washed with water $(10 \mathrm{~mL})$, brine $(10 \mathrm{~mL})$, dried over anhydrous $\mathrm{Na}_{2} \mathrm{SO}_{4}$, and evaporated under reduced pressure. The residue was purified by column chromatography to afford the desired product.

The isolated yields of pure compounds obtained from this procedure are reported in Table 1 . The following compounds were displayed identical spectroscopic properties to authentic samples: $\mathbf{1 b}, \mathbf{2} \mathbf{b}, \mathbf{3 b}$, $4 b, 5 b, 6 b, 7 b, 12 b, 13 b, 17 b$.

2-Allyl-4-methoxy-phenol (9b): the general deprotection procedure was applied on 9a to afford $9 \mathbf{b}$ (98 $\%)$ as a colorless oil. ${ }^{1} \mathrm{H}$ NMR $\left(\mathrm{CDCl}_{3} ; 200 \mathrm{MHz}\right): \delta 3.32-3.60\left(\mathrm{~m}, 2 \mathrm{H}, \mathrm{Ar}-\mathrm{CH}_{2}-\right) ; 3.79\left(\mathrm{~s}, 3 \mathrm{H},-\mathrm{OCH}_{3}\right)$; 5.10-5.24 (m, 2H, $\left.-\mathrm{CH}=\mathrm{CH}_{2}\right) ; 5.40$ (brs., $\left.1 \mathrm{H},-\mathrm{OH}\right) ; 5.90-6.16\left(\mathrm{~m}, 1 \mathrm{H},-\mathrm{CH}=\mathrm{CH}_{2}\right) ; 6.60-6.90(\mathrm{~m}, 3 \mathrm{H}$, Aryl); ${ }^{13} \mathrm{C}$ NMR $\left(\mathrm{CDCl}_{3}\right) \delta 34.7,55.5,112.3,114.6,115.7,116.1,126.6,135.9,147.6,153.3$; IR (thin film, $\left.\mathrm{cm}^{-1}\right): 3333$ (b, OH), 2834, 1820, $1606(-\mathrm{C}=\mathrm{C}-), 1216,910,737$; ESMS $\mathrm{m} / z$ (relative intensity) 183 $(\mathrm{M}+1)^{+}(16), 121$ (100); HRMS calcd for $\mathrm{C}_{10} \mathrm{H}_{13} \mathrm{O}_{2}$ 165.0910, found 165.0907.

2-Allyl-4-prop-2-ynyloxy-phenol (10b): the general deprotection procedure was applied to 10a to afford the Claisen-rearranged product $\mathbf{1 0 b}(84 \%)$ as a colorless oil. ${ }^{1} \mathrm{H}$ NMR $\left(\mathrm{CDCl}_{3} ; 200 \mathrm{MHz}\right): \delta 2.51(\mathrm{t}, J=$ $2.2 \mathrm{~Hz}, 1 \mathrm{H},-\mathrm{CCH}) ; 3.39\left(\mathrm{dt}, J=5.8\right.$ and $\left.1.6 \mathrm{~Hz}, 2 \mathrm{H}, \mathrm{Ar}_{-} \mathrm{CH}_{2-}\right) ; 4.63\left(\mathrm{~d}, J=2.2 \mathrm{~Hz}, 2 \mathrm{H},-\mathrm{CH}_{2}-\mathrm{CC}\right) ; 4.86$ (brs., $1 \mathrm{H},-\mathrm{OH}) ; 5.10-5.24\left(\mathrm{~m}, 2 \mathrm{H},-\mathrm{CH}=\mathrm{CH}_{2}\right) ; 5.90-6.12\left(\mathrm{~m}, 1 \mathrm{H},-\mathrm{CH}=\mathrm{CH}_{2}\right) ; 6.74-6.82(\mathrm{~m}, 3 \mathrm{H}$, Aryl); ${ }^{13} \mathrm{C}$ NMR $\left(\mathrm{CDCl}_{3}\right) \delta 34.5,55.9,74.6,78.2,113.3,115.7,116.0,116.7,125.9,135.3,148.0,151.0$; IR (thin film, $\mathrm{cm}^{-1}$ ): 3400 (b, OH), 3289 (HCC-), 2910, 2114 (alkyne), 1832, 1602 (-C=C-), 1200, 906; GCMS $m / z 188(\mathrm{M}), 149$.

4-Prop-2-ynyloxy-phenol (11b): the general deprotection procedure was applied on 11 a to afford 11b $(61 \%)$ as a colorless oil. ${ }^{1} \mathrm{H}$ NMR $\left(\mathrm{CDCl}_{3} ; 200 \mathrm{MHz}\right): \delta 2.51$ (t, $\left.J=2.4,1 \mathrm{H},-\mathrm{CCH}\right) ; 4.62$ (d, J = 2.4 Hz, $\left.2 \mathrm{H},-\mathrm{CH}_{2}-\mathrm{CC}\right) ; 5.66$ (brs., $\left.1 \mathrm{H},-\mathrm{OH}\right) ; 6.72-6.92\left(\mathrm{~m}, 4 \mathrm{H}\right.$, Aryl); ${ }^{13} \mathrm{C} \mathrm{NMR}\left(\mathrm{CDCl}_{3}\right) \delta$ 56.4, 75.0, 78.5, 115.7, 116.0, 150.1, 151.3; IR (thin film, $\mathrm{cm}^{-1}$ ): 3350 (b, -OH), 3289 (HCC-), 2117 (alkyne), 1501, 1445, 1195, 1026, 821; GCMS m/z 148 (M), 109.

4-Hydroxy- $N$-prop-2-ynyl-benzamide (14b): the general deprotection procedure was applied to 14a using 3.0 eq. $\mathrm{BBr}_{3}$ to afford $\mathbf{1 4 b}(35 \%)$ as a colorless oil. ${ }^{1} \mathrm{H}$ NMR $\left(\mathrm{CDCl}_{3} ; 200 \mathrm{MHz}\right): \delta 2.56(\mathrm{t}, J=2.2$ $\mathrm{Hz}, 1 \mathrm{H},-\mathrm{CCH}) ; 4.12-4.18\left(\mathrm{~m}, 2 \mathrm{H},-\mathrm{CH}_{2}-\mathrm{CC}\right) ; 6.81$ (d, $J=8.6 \mathrm{~Hz}, 2 \mathrm{H}$, Aryl); 7.69 (d, $J=8.6 \mathrm{~Hz}, 2 \mathrm{H}$, Aryl); 8.50-8.65 (m, 1H, -NH); ${ }^{13} \mathrm{C}$ NMR (CD $\left.{ }_{3} \mathrm{OD}\right) \delta 26.6,67.0,72.0,81.1,114.8,116.2,127.2,130.4$, 162.1, 164.1; IR (thin film, $\mathrm{cm}^{-1}$ ): 3293 (b, -OH and HCC-), 2938, 1602 (-C=O), 1276, 1231, 1175, 1046, 
921, 845, 760; ESMS $m / z$ (relative intensity) $198(\mathrm{M}+\mathrm{Na})^{+}(9), 176(\mathrm{M}+1)^{+}(7)$; HRMS calcd for $\mathrm{C}_{10} \mathrm{H}_{10} \mathrm{NO}_{2}$ 176.0706, found 176.0706.

4-Hydroxy- $\mathrm{N}$-methyl- $\mathrm{N}$-prop-2-ynyl-benzamide (15b): the general deprotection procedure was applied on 15a using 2.0 eq. $\mathrm{BBr}_{3}$ to afford 15b (41\%) as a colorless oil. ${ }^{1} \mathrm{H}$ NMR $\left(\mathrm{CDCl}_{3} ; 200 \mathrm{MHz}\right): \delta 2.77$ (brs., $1 \mathrm{H},-\mathrm{CCH}) ; 3.10$ (s, 3H, -N-CH $) ; 4.21$ (brs., $\left.2 \mathrm{H},-\mathrm{CH}_{2}-\mathrm{CC}\right) ; 6.83$ (d, $J=8.4 \mathrm{~Hz}, 2 \mathrm{H}$, Aryl); 7.36 (d, $J=8.4 \mathrm{~Hz}, 2 \mathrm{H}$, Aryl); IR (thin film, cm ${ }^{-1}$ ): 3289 (b, -OH and HCC-), 2802, 2114 (alkyne), 1605 (-C=O), $1272,1232,1168,1067,934,846,757$; ESMS $m / z$ (relative intensity) $212(\mathrm{M}+\mathrm{Na})^{+}(10), 190(\mathrm{M}+1)^{+}$ (30); HRMS calcd for $\mathrm{C}_{11} \mathrm{H}_{11} \mathrm{NO}_{2}$ 188.0717, found 188.0715 .

2-Chloro- $N$-(3-hydroxy-5-prop-2-ynyloxy-benzyl)-terephthalamic acid methyl ester (16b): the general deprotection procedure was applied to $\mathbf{1 6 a}$ using 2.0 eq. $\mathrm{BBr}_{3}$ to afford $\mathbf{1 6 b}(71 \%)$ as a colorless oil. ${ }^{1} \mathrm{H}$ NMR $\left(\mathrm{CDCl}_{3} ; 200 \mathrm{MHz}\right): \delta 2.89(\mathrm{t}, J=2.4 \mathrm{~Hz}, 1 \mathrm{H},-\mathrm{CCH}) ; 3.92\left(\mathrm{~s}, 3 \mathrm{H},-\mathrm{OCH}_{3}\right) ; 4.46-4.60(\mathrm{~m}$, $\left.2 \mathrm{H},-\mathrm{CH}_{2}-\mathrm{N}\right) ; 4.65\left(\mathrm{~d}, J=2.4 \mathrm{~Hz}, 2 \mathrm{H},-\mathrm{CH}_{2}-\mathrm{CCH}\right) ; 6.30-6.36(\mathrm{~m}, 1 \mathrm{H}$, Aryl); 6.40-6.48 (m, $2 \mathrm{H}$, Aryl); 7.76-7.90 (m, 2H, Aryl); 7.94-7.98 (m, 1H, Aryl); 9.09 (brt., J = 5.2 Hz, 1H, -NH); ). IR (thin film, $\mathrm{cm}^{-1}$ ): 3289 (-OH, HCC-), 2955, 2126 (alkyne), 1961, 1723 (-C=O), 1635, 1595, 1542, 1333, 1297, 1244, 1150, $1047,842,750$.

3,5-Dimethyl-4-prop-2-ynyloxy-phenol (19b): the general deprotection procedure was applied to 19a to afford 19b $(55 \%)$ as white solid. Mp $72-73{ }^{\circ} \mathrm{C} .{ }^{1} \mathrm{H} \mathrm{NMR}\left(\mathrm{CDCl}_{3}\right) \delta 2.23\left(\mathrm{~s}, 6 \mathrm{H}, \mathrm{Ar}-\mathrm{CH}_{3}\right), 2.50(\mathrm{t}, J=2.4$ $\mathrm{Hz}, 1 \mathrm{H},-\mathrm{CCH}), 4.46(\mathrm{~s}, 1 \mathrm{H}, \mathrm{Ar}-\mathrm{OH}), 4.61\left(\mathrm{~d}, \mathrm{~J}=2.4 \mathrm{~Hz}, 2 \mathrm{H},-\mathrm{CH}_{2}-\mathrm{CC}\right), 6.63$ (s, 2H, Aryl); ${ }^{13} \mathrm{C}$ NMR $\left(\mathrm{CDCl}_{3}\right) \delta$ 15.6, 55.8, 74.5, 78.4, 114.5, 123.5, 146.2, 150.2; IR $\left(\mathrm{KBr}, \mathrm{cm}^{-1}\right): 3405$ (b, HCC-, -OH), 3309, 2931, 2351, 2126 (alkyne), 1647, 1345, 1196, 1095, 826; ESMS $m / z$ (relative intensity) $199(\mathrm{M}+\mathrm{Na})^{+}$ (30), $177(\mathrm{M}+1)^{+}(80)$; HRMS calcd for $\mathrm{C}_{11} \mathrm{H}_{13} \mathrm{O}_{2}$ 177.091, found 177.091.

The regiochemistry of deprotection was confirmed by the sequence of reactions shown below. Allylation was followed by rapid $\mathrm{BBr}_{3}$-induced Claisen rearrangement to afford $\mathbf{2 3}$ in high yield. Such a rearrangement would not have been possible with the other potential regioisomer of $\mathbf{2 2}$, since the ortho and para positions would be blocked.<smiles>C#CCOc1c(C)cc(O)cc1C</smiles>

$19 b$

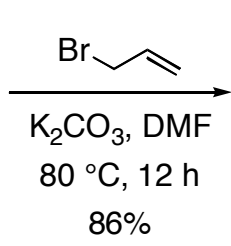

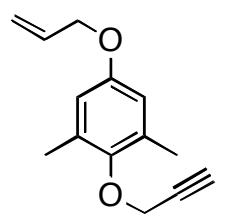

22

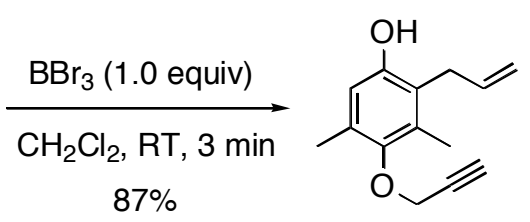

23

5-Allyloxy-1,3-dimethyl-2-prop-2-ynyloxy-benzene (22): the general protection procedure was applied to $19 \mathrm{~b}$ using allyl bromide to afford $22(86 \%)$ as colorless oil. ${ }^{1} \mathrm{H}$ NMR $\left(\mathrm{CDCl}_{3}\right) \delta 2.27\left(\mathrm{~s}, 6 \mathrm{H}, \mathrm{Ar}-\mathrm{CH}_{3}\right)$, $2.51(\mathrm{t}, J=2.0 \mathrm{~Hz}, 1 \mathrm{H},-\mathrm{CCH}), 4.26\left(\mathrm{~d}, J=5.6 \mathrm{~Hz}, 2 \mathrm{H},-\mathrm{CH}_{2}-\right), 4.63\left(\mathrm{~d}, J=1.8 \mathrm{~Hz}, 2 \mathrm{H},-\mathrm{CH}_{2}-\mathrm{CC}\right), 5.24-$ $5.47\left(\mathrm{~m}, 2 \mathrm{H},-\mathrm{HC}=\mathrm{CH}_{2}\right), 6.01-6.21\left(\mathrm{~m}, 1 \mathrm{H},-\mathrm{CH}=\mathrm{CH}_{2}\right), 6.63(\mathrm{~s}, 2 \mathrm{H}, \mathrm{Aryl}) ;{ }^{13} \mathrm{C} \mathrm{NMR}\left(\mathrm{CDCl}_{3}\right) \delta 16.5$, 55.8, 73.1, 75.1, 78.7, 114.5, 116.9, 131.8, 134.0, 150.2, 153.1; IR (thin film, $\mathrm{cm}^{-1}$ ): 3293 (HCC-), 3087, 3023 (-C=C-H), 2927, 2866, 2363, 2335, 2126 (alkyne), 1719, 1651 (-C=C-), 1598, 1482, 1208, 1059, 987, 793; ESMS m/z (relative intensity) $239(\mathrm{M}+\mathrm{Na})^{+}(100), 149(50), 102(43)$.

2-Allyl-3,5-dimethyl-4-prop-2-ynyloxy-phenol (23): the general deprotection procedure was applied to compound 22 to afford Claisen-rearranged product $23(87 \%)$ as a white solid. Mp 73-74 ${ }^{\circ} \mathrm{C} ;{ }^{1} \mathrm{H}$ NMR $\left(\mathrm{CDCl}_{3}\right) \delta 2.17,2.24(2 \mathrm{~s}, 6 \mathrm{H}, \mathrm{Ar}-\mathrm{CH} 3), 2.46(\mathrm{t}, J=2.41 \mathrm{~Hz}, 1 \mathrm{H},-\mathrm{CCH}), 3.42\left(\mathrm{~d}, J=5.62 \mathrm{~Hz},-\mathrm{CH}_{2}-\right)$, $4.33(\mathrm{~s}, 1 \mathrm{H}, \mathrm{Ar}-\mathrm{OH}), 4.62\left(\mathrm{~d}, J=2.41 \mathrm{~Hz},-\mathrm{CH}_{2}-\mathrm{CC}\right), 4.86-4.99\left(\mathrm{~m}, 2 \mathrm{H},-\mathrm{CH}_{2}=\mathrm{CH}-\right), 5.83-5.97(\mathrm{~m}, 1 \mathrm{H},-$ $\left.\mathrm{CH}_{2}=\mathrm{CH}-\right), 6.68(\mathrm{~s}, 1 \mathrm{H}, \mathrm{Aryl}) ;{ }^{13} \mathrm{C} \mathrm{NMR}\left(\mathrm{CDCl}_{3}\right) \delta 11.9,16.4,30.6,31.0,57.4,74.9,78.1,113.6,114.6$, 121.0, 123.7, 136.4, 149.3; IR (KBr, cm $\left.{ }^{-1}\right): 3417$ (b, HCC-, -OH), 3289, 3091, 2931, 2870, 2118 (alkyne), $1675(-\mathrm{C}=\mathrm{C}-), 1478,1305,1212,1111,910,838$; ESMS $m / z$ (relative intensity) $239(\mathrm{M}+\mathrm{Na})^{+}(27), 217$ $(\mathrm{M}+1)^{+}(17)$. 
4-Methoxy-2,6-dimethyl-phenol (20b): the general deprotection method was applied to compound 20a

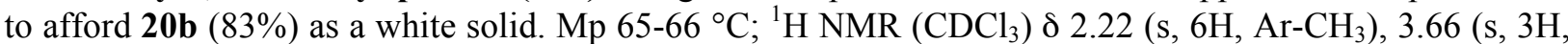

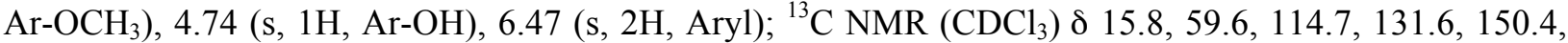
150.9; IR (KBr, $\left.\mathrm{cm}^{-1}\right): 3510$ (b, -OH), 2667, 2351, 2162, 1606, 1478, 1321, 1216, 1007, 858; ESMS m/z (relative intensity) $151(\mathrm{M}-1)^{-}(13), 113$ (100).

The regiochemistry of $\mathbf{2 0 b}$ was determined from $\mathbf{1 9 b}$ by the sequence shown here:<smiles>Cc1cc(O)cc2c1OCC#C2</smiles>

$19 b$

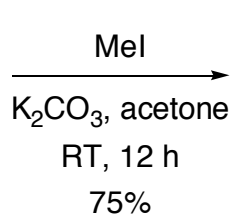

$75 \%$<smiles>C=CCOc1c(C)cc(OC)cc1C</smiles>

20a

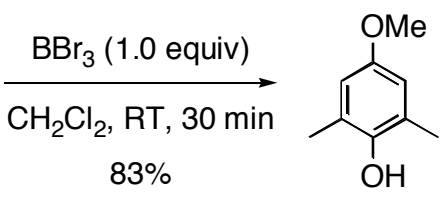

20b

3,5-Di-tert-butyl-4-prop-2-ynyloxy-phenol (21b): the general deprotection procedure was applied to 21a to afford $21 \mathbf{b}(68 \%)$ as a colorless oil. ${ }^{1} \mathrm{H}$ NMR $\left(\mathrm{CDCl}_{3}\right) \delta 1.35\left(\mathrm{~s}, 18 \mathrm{H},-\mathrm{C}\left(\mathrm{CH}_{3}\right)_{3}\right), 2.43(\mathrm{t}, J=2.1$ $\mathrm{Hz}, 1 \mathrm{H},-\mathrm{CCH}$ ), 4.35 (d, $J=2.1 \mathrm{~Hz},-\mathrm{CH}_{2}-\mathrm{CC}$ ), 5.22 (br s., $\left.1 \mathrm{H}, \mathrm{Ar}-\mathrm{OH}\right), 6.74$ (s, $2 \mathrm{H}$, Aryl); ${ }^{13} \mathrm{C}$ NMR $\left(\mathrm{CDCl}_{3}\right) \delta 31.6,35.6,79.2,113.3,118.4,137.1,144.4,149.2,150.6$; IR (thin film, $\left.\mathrm{cm}^{-1}\right): 33305$ (HCC-, OH), 2967, 2906, 2870, 2254 (alkyne), 1715, 1639, 1586, 1325, 1204, 1003, 962, 854; ESMS m/z (relative intensity) $261(\mathrm{M}+1)^{+}(80), 257(20)$; HRMS calcd for $\mathrm{C}_{17} \mathrm{H}_{25} \mathrm{O}_{2} 261.1855$, found 261.1313.

\section{Examination of reaction mixture by deuterium NMR}

Monodeuterated 1a was prepared and its reaction with a slight deficiency of $\mathrm{BBr}_{3}$ at room temperature in $\mathrm{CH}_{2} \mathrm{Cl}_{2}$ (containing $5 \% \mathrm{CDCl}_{3}$ for reference and spectrometer locking) was examined by ${ }^{2} \mathrm{H} \mathrm{NMR}$, as shown in Figure $\mathrm{S} 1$. 
Figure S1. ${ }^{2} \mathrm{D}$ NMR of a the deprotection reaction of $1 \mathrm{a}-d_{1}$. Bromoallene is expected to give a resonance at approximately $5.9 \mathrm{ppm}$.
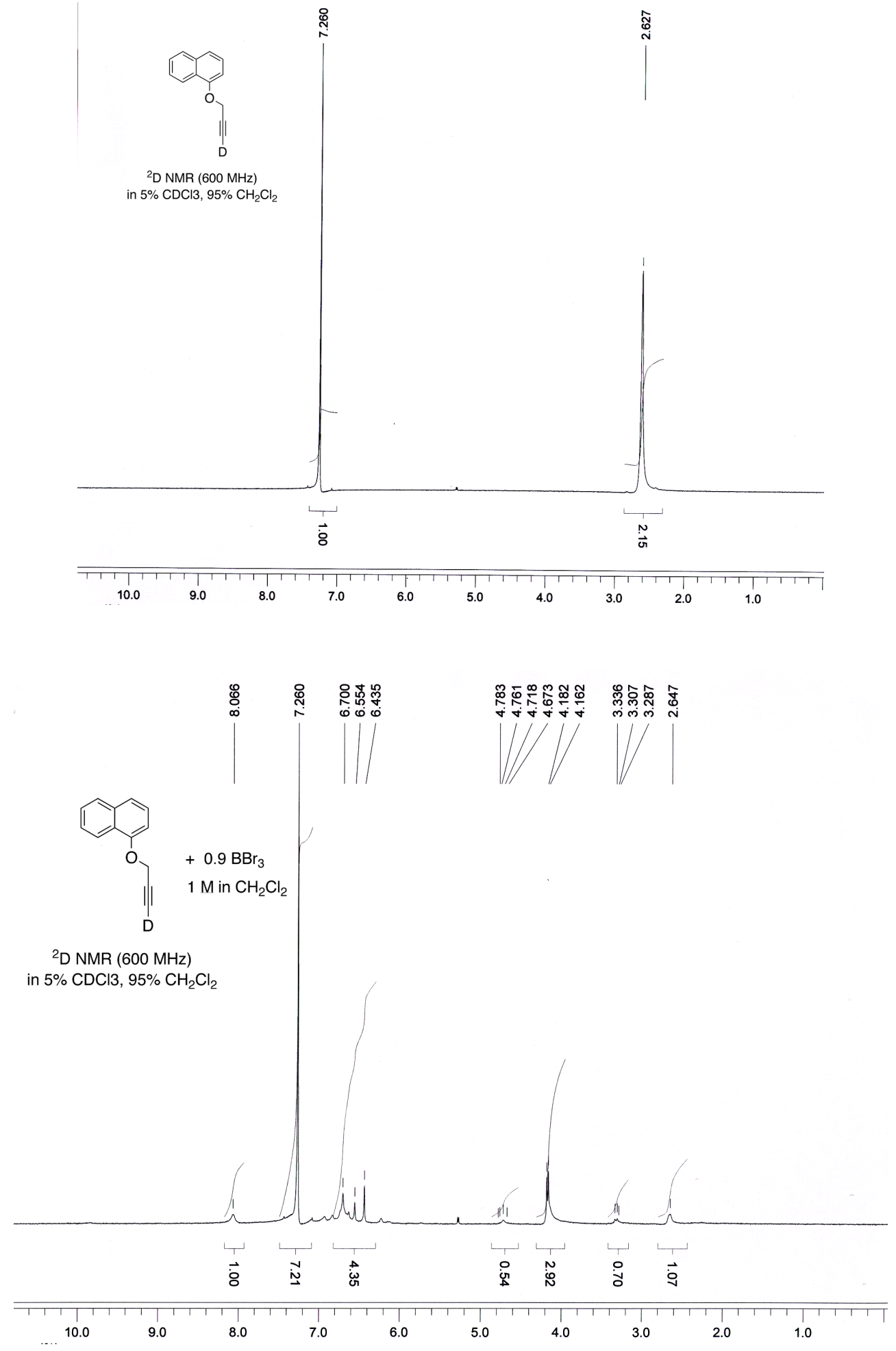